\title{
Quantifying Staphylococcus Aureus Biofilm Formation on Stainless Steel and Titanium Alloy Used in Orthopedic Fracture Fixation
}

Shayesteh Beladi-Behbahani

Clemson University

\section{Sarah M. Helms}

Clemson University

John D. DesJardins

Clemson University

Marian S. Kennedy

Clemson University

Terri Bruce

Clemson University

Tzuen-Rong J. Tzeng ( $\nabla$ tzuenrt@clemson.edu )

Clemson University

\section{Research Article}

Keywords: Biofilm, Staphylococcus aureus, orthopedic implants, fracture fixation, infection

Posted Date: March 1st, 2021

DOl: https://doi.org/10.21203/rs.3.rs-253531/v1

License: (1) This work is licensed under a Creative Commons Attribution 4.0 International License.

Read Full License 
$12 *$ Corresponding author

Email: tzuenrt@clemson.edu

Phone: 864.656.0239

\section{ABSTRACT}

\section{Quantifying Staphylococcus aureus biofilm formation on stainless steel and titanium alloy used in} orthopedic fracture fixation

\section{S. Beladi-Behbahani ${ }^{1}$, S. M. Helms ${ }^{2}$, J. D. DesJardins ${ }^{2}$, M. S. Kennedy ${ }^{3}$, T. Bruce ${ }^{4}$, T-R J. Tzeng ${ }^{1}$}

${ }^{1}$ Department of Biological Sciences, Clemson University, Clemson, SC

${ }^{2}$ Department of Bioengineering, Clemson University, Clemson, SC

${ }^{3}$ Department of Materials Science and Engineering, Clemson University, Clemson, SC

${ }^{4}$ Clemson Light Imaging Facility, Clemson, SC

Running Title: Quantification of biofilms on implant surfaces

\section{Dr. Tzuen-Rong Jeremy Tzeng}

Address: 190 Collings Street, 149 Life Sciences Facility, Clemson, SC 29634

In order to assess and compare the antibacterial property of implants surfaces, a standard method is needed to quantify bacterial load. This study evaluated the effectiveness of three quantifying methods, namely, (I) crystal violet staining analysis, (II) ultrasound detachment with viable cell counts, and (III) confocal laser scanning microscopy for characterizing S. aureus Seattle 1945 (ATCC 25923) biofilm on metallic coupons. The accuracy of the results, time for completion, and ease of use of methods were compared. The crystal violet method is relatively faster and more straightforward for analyzing biofilm formation. However, the accuracy of the confocal laser scanning microscopy method is found to be 
considerably higher than that of the other methods. Confocal laser scanning microscopy method is

27 considered to be more time-consuming for data collection and analysis and costlier. The ultrasound

28 detachment followed by viable cell count of recovered cells is recommended for biofilm quantification

29 analysis on orthopedic materials when there is a large number of samples (more than ten samples). This

30 info could provide guidelines that would facilitate the selection of suitable method for quantifying biofilm

31 formation on orthopedic implants based on investigators' consideration on method accessibility, assay cost, 32 assay time, and complexity of method.

34 Keywords: Biofilm, Staphylococcus aureus, orthopedic implants, fracture fixation, infection

INTRODUCTION

Biofilms consist of a group of microorganisms attached to a surface within an extracellular polymeric substance matrix. When the film structure is established, the microorganisms inside it are more resistant to various antimicrobial agents ${ }^{1}$. Post-surgery biofilm formation on orthopedic implants is a major problem in the treatment of the patients ${ }^{2} \mathrm{~A}$ biofilm delays the healing process and increases the probability

41 that revision surgeries may be needed to remove the contaminated implant ${ }^{2}$. The attachment of the biofilm

42 has been shown to be a complex process that is influenced by different environmental factors and surface

43 characteristics ${ }^{3}$. Surface roughness, and associated changes in effective surface area, is one of the known

44 factors that affect microorganism's colonization. It appears that colonization increases with escalations in 45 surface roughness ${ }^{4}$. In addition to roughness, surface hydrophobicity can also play a role in the initial 46 attachment. Some studies showed that microorganisms attach faster to hydrophobic, nonpolar surfaces such 47 as Teflon than hydrophilic surfaces like glass or metals ${ }^{3,4}$.

48 New implant materials or surface modification methods have been developed to reduce biofilm 49 formation ${ }^{5-7}$. Prior to in vivo application of these materials or surface modifications, the anti-biofilm 50 properties need to be evaluated in vitro. Currently, there is a range of methods that have been used to 51 quantify biofilms on solid surfaces in vitro and these can be classified into three groups: (I) microscopic 
52 methods, (II) bacterial culture-based procedures, and (III) biochemical investigations ${ }^{8}$. However, 53 researchers need to consider the limitations and accuracy of each method prior to their application of these 54 within a study. While there have been some comparison reviews that sought to rank these techniques, there 55 is a need to compare the benefits to employing these techniques for larger scale, specifically for orthopedic 56 modified surfaces.

57 In this study, three different quantification methods were compared for characterization of biofilm

58 formed on the surfaces of stainless steel and titanium disks, two of the most commonly used alloys in 59 fracture fixation implants ${ }^{9}$. Based on the experimental needs of accessibility and the initial estimates of cost 60 and required time for each method, crystal violet (CV) staining, confocal laser scanning microscopy 61 (CLSM) and sonication with viable cell count (VCC) were selected for evaluation. Crystal violet staining, a low cost, simple and fast method for analysis of biofilm, was first described by Christensen et al. $1985^{10}$. CV is a basic protein dye that binds to negatively charged surface

64 molecules and polysaccharides in the extracellular matrix ${ }^{11}$. The method is basically based on staining the biofilm formed on a surface with $\mathrm{CV}$, then rinsed a few times to remove non-attached bacterial cells, washed with $95 \% \mathrm{v} / \mathrm{v}$ ethanol to release the bound $\mathrm{CV}$ and then the optical density (OD) is recorded with a

67 spectrometer. The concentration of $\mathrm{CV}$ recovered is considered to be proportional to the amount of biofilm 68 on metal surfaces ${ }^{12}$. It should be noted that CV stains both live cells, dead cells and their matrix and 69 therefore, it can not be used to evaluate a functional biofilm ${ }^{13}$.

CLSM is a technique for capturing high-resolution optical images at different depths. As it can 71 provide depth sectioning of thick specimens, it is a suitable method for analyzing thickness, biomass and 72 structure through the three-dimensional (3D) images or cross-sectional views of the biofilm. It has been 73 used extensively to increase the understanding of biofilm characteristics ${ }^{14}$. Development of new analytical 74 methods and software increased the quantitative output of these images ${ }^{14}$. An automatic threshold can be 75 used in MATLAB to separate the bacteria and surface sequences ${ }^{15,16}$.

76 Ultrasound detachment combined with viable cell count is another method used to analyze biofilm

77 formed on surfaces. It is more complicated and time-consuming than the CV method, but less costly and 
78 faster than laser confocal microscopy ${ }^{17,18}$. The method is based on recovering the live bacteria from the 79 surface of biofilm by detaching them through a sonication technique followed by a plate counting $\operatorname{method}^{8}$.

80 The low-moderate sonication $(20-40 \mathrm{kHz})$ of the bacterial suspension in the water bath for a short duration

81 (2-10 $\mathrm{min})$, has been shown to have the optimum effect on bacterial detachment. Longer exposure time or 82 higher ultrasound power leads to disruption of bacterial cell wall ${ }^{19,20}$.

83 In this study, three different quantification methods were compared for the characterization of 84 biofilm formed on the surfaces of stainless steel and titanium disks. A fluorescent strain of Staphylococcus 85 aureus (S. aureus) expressing intracellular green fluorescent protein (GFP) was used to facilitate the 86 observation of colonization and biofilm formation on metal coupons via CV staining, laser confocal 87 microscopy and viable cell count methods. Protocols for cultivation and quantification of biofilms on stainless steel and titanium alloys surfaces were developed and validated.

MATERIALS AND METHODS

\section{Titanium and stainless steel cylindrical coupons}

Stainless steel and titanium are the most common alloys used for fracture fixation implants ${ }^{9}$. In this

94 study, biofilms were grown on titanium-6Al-4Vand 316 stainless steel cylindrical disks (Lisnabrin 95 Engineering, Ireland) and will be referred to in this manuscript as Ti and SS coupons respectively. Each 96 coupon ( $1 \mathrm{~cm}$ diameter, $1 \mathrm{~mm}$ thickness) were laser cut from $1 \mathrm{~mm}$ thick sheets of either grade $5 \mathrm{Ti}-6 \mathrm{Al}-$ $974 \mathrm{~V}$ or $316 \mathrm{SS}$ and no additional steps were taken to polish their respective surfaces. Surface roughness (Ra) 98 of Ti $(n=30)$ and SS $(n=30)$ was measured in six randomized locations on each sample. The average 99 surface roughness of Ti coupons was $0.56 \mu \mathrm{m}(\mathrm{SD}=0.10 \mu \mathrm{m})$ and $\mathrm{SS}$ coupons was $0.20 \mu \mathrm{m}(\mathrm{SD}=0.01$ $100 \mu \mathrm{m})$.

101

\section{Bacterial strain}


For quantifying the growth of the biofilm on the SS and Ti coupons, the S. aureus Seattle 1945 strain (ATCC 25923) was used. It is a clinical isolate often used as a standard laboratory testing control $^{21-}$

$105{ }^{23}$. This bacterium was transformed with plasmid pGFPuvr, which encodes green fluorescent proteins. Thus, 106 the presence, growth and colonization of bacteria can be evaluated using fluorescent microscopy ${ }^{24,25}$.

\section{Bacterial growth on the coupons}

S. aureus stock cultures were streaked onto the tryptic soy agar (TSA) $+1 \%$ glucose plates and incubated overnight at $37^{\circ} \mathrm{C}$ to obtain isolated colonies. A single colony was then transferred onto tryptic soy broth (TSB) media and grown overnight in a shaker incubator at $37^{\circ} \mathrm{C}$ and $125 \mathrm{rpm}$ to the concentration around $1 \times 10^{9} \mathrm{CFU} / \mathrm{mL}$ The overnight cultures were then used to inoculate the experimental coupons.

\section{Coupon cleaning procedure}

Prior to use, coupons were cleaned and sterilized by soaking in a $70 \% \mathrm{v} / \mathrm{v}$ ethanol solution for 10 min, rinsed in 1x PBS $(0.01 \mathrm{M})$ solution, then soaked in ethanol solution and finally rinsed with PBS and

117 air dried in sterile petri dishes.

\section{Coating the underside surface of the coupons with VaLaP}

To be able to quantify the amount of biofilm formed on just the upper side of the SS and Ti coupons,

121 the underside of individual coupons was coated a mixture of vaseline, lanolin and paraffin wax in equal 122 rations (VaLaP). This is a biologically inert material that has been shown to prevent bacterial attachment ${ }^{26}$. 123 Covering one side of the surface is important when quantifying biofilm using the CV and ultrasound with 124 VCC methods, while it is not necessary when using the CLSM method as only the upper surface is imaged.

\section{Cultivation and quantification of biofilm}

Cultivation of biofilm growth were conducted using the following protocol. Briefly, 24-well flatbottom non-tissue-culture treated microtiter plates were used to minimize binding of bacteria onto the wells 
and to facilitate the cultivation of biofilm on metal coupon surfaces. Coupons were dipped gently into melted VaLaP until the bottom side was covered. Each coupon was then placed into a separate well of the 24-well plate. $600 \mu \mathrm{L}$ of TSB $+1 \%$ glucose was added to each well to cover the entire surface of the coupon.

132 The concentration of the overnight $S$. aureus was determined spectrophotomically and $10^{3}$ bacterial cells 133 were added to each well containing coupon and the plate was incubated at $37^{\circ} \mathrm{C}$ for $24 \mathrm{~h}$. Washing of metal 134 coupons after growth of biofilms was carried out by dipping the coupons in wells containing $500 \mu \mathrm{L}$ of 135 deionized (D.I.) water. This process was repeated three times to wash away planktonic bacteria. The 136 biofilms on metal coupons were then observed using an epi-fluorescence microscope or a laser confocal 137 microscope. For quantification of biofilm, the CV staining method or viable cell count experiment was 138 used. A positive control, i.e., a well containing TSB and bacterial inoculum, and a negative control, i.e., a 139 well containing TSB without bacteria were included for each individual experiment ${ }^{14,16}$.

\section{Quantification of biofilm via CV staining}

For quantification of biofilm growth on the coupons using CV method, they were processed as follows: After the last wash to remove unbound planktonic cells, the coupons were placed into empty wells 144 and allowed to air-dry at $37^{\circ} \mathrm{C}$ for $30 \mathrm{~min}$. Metal coupons were placed into wells containing $600 \mu \mathrm{L}$ of $1450.1 \%(\mathrm{w} / \mathrm{v})$ aqueous $\mathrm{CV}$ solution and stained for $5 \mathrm{~min}$ at room temperature $\left(22^{\circ} \mathrm{C}\right)$. The metal coupons 146 were then washed three times in wells containing $600 \mu \mathrm{L}$ of D.I. to remove excess stains and air-dried at $14737^{\circ} \mathrm{C}$ for $30 \mathrm{~min} .600 \mu \mathrm{L}$ of $100 \%$ ethanol was added to the wells containing coupons to dissolve the bound $148 \mathrm{CV}$ and the plate was placed on a shaker $(50 \mathrm{rpm})$ at room temperature for $10 \mathrm{~min}$ to detach the adherent 149 biofilm growing on the coupons. $100 \mu \mathrm{L}$ of each solution was transferred into a 96-well microtiter plate 150 and the biofilm quantified using a Synergy H1 Hybrid Microplate Reader reading at $590 \mathrm{~nm}^{12}$. 
The fluorescence property of S. aureus Seattle $1945^{\mathrm{GFPuvr}}$ strain was verified using a Zeiss laser

154 confocal microscope. CLSM is a technique for obtaining high-resolution optical images with depth 155 selectivity (Z-stacks). The main feature of confocal microscopy is its ability to acquire in-focus images

156 from selected depths. Washing metal coupons after the growth of biofilms is completed by dipping the 157 coupons in wells containing $600 \mu \mathrm{L}$ of D.I. water. This process is repeated three times to wash away 158 planktonic bacteria. The biofilms on metal coupons were then observed using a laser confocal microscope. 159 Images were acquired point-by-point and reconstructed with the computer, allowing 3D reconstructions of 160 topologically complex objects. 3D images enable us to exam biofilm structure on different coupons. 161 Interpreting the images through measuring some features of bacterial biofilm using COMSTAT gave us the 162 ability to determine how the biofilm formed on different surfaces ${ }^{15,16}$.

\section{Biofilm thickness and biomass analysis}

To analyze the average thickness and biomass of biofilm formed on coupon surfaces, multiple laser confocal image stacks were analyzed using the COMSTAT program. The image acquisition and data 167 analysis were standardized and optimized, e.g., size of inoculum, incubation time, and the number of stacks.

168 There are some features that are calculated by COMSTAT including Bio-volume, which is defined as the 169 number of biomass pixels in all images of a stack multiplied by the voxel size [(pixel size $)_{\mathrm{x}} \times(\text { pixel size })_{\mathrm{y}}$ $\left.170 \times(\text { pixel size })_{z}\right]$ and divided by the substratum area of the image stack. The resulting value is biomass volume 171 divided by substratum area $\left(\mu \mathrm{m}^{3} / \mu \mathrm{m}^{2}\right)$. Bio-volume represents the overall volume of the biofilm and 172 provides an estimate of the biomass in the biofilm. Area occupied by bacteria in each layer was fraction of 173 the area occupied by biomass in each image of a stack and the substratum coverage is the area coverage in 174 the first image of the stack. Substratum coverage reflects how efficient the colonization of bacteria is on 175 the substratum. Thickness distribution and mean thickness: This function locates the highest point $(\mu \mathrm{m})$ 176 above each (x, y) pixel in the bottom layer containing biomass. Hence, the thickness is defined as the 177 maximum thickness over a given location, ignoring pores and voids inside the biofilm. Mean biofilm 
thickness provides a measure of the spatial size of the biofilm and is the most common variable used in biofilm literature ${ }^{27}$.

\section{Quantification of biofilm using viable cell count}

A viable count test was performed to quantify biofilm formation on the coupons since this method yields more consistent and sensitive results than the CV method. We followed the Bjerkan method with modification; briefly, after cultivation of biofilm on the coupon surface, each disk was placed into a $15 \mathrm{~mL}$ sterile conical tube containing $5 \mathrm{~mL}$ of PBS and gently vortexed for 15 seconds to rinse off planktonic cells. After addition of $5 \mathrm{~mL}$ of PBS, each tube was then subjected to sonication for $5 \mathrm{~min}$ at full wave (40 KHz) in a DK Sonic water bath ultra-sonic cleaner. The power was enough to detach the bacterial cells from the surface, but not to damage the cells. This wash 1 solution (W1) was serially diluted $10^{-1}, 10^{-2}, 10^{-3}$, and $10^{-}$

${ }^{4}$ folds. $100 \mu \mathrm{L}$ from each undiluted and diluted sample was plated on TSA and incubated at $37{ }^{\circ} \mathrm{C}$ for 48 h. Each disk was washed a second time in $5 \mathrm{~mL}$ PBS in ultrasound water bath for another 5 min yielding the second wash solution (W2). The washing process was repeated for the third time and generated a third wash solution (W3). Viable counts of W2 and W3 was conducted the same way described for W1. Numbers of colonies were counted and the total cell number recovered from each coupon was calculated ${ }^{19}$.

\section{Validation of the methods using glass cover slips}

To validate the biofilm quantifying methods, biofilms grown on round glass coverslips (Prod No. 260368, Ted Pella INC) with a diameter of $10 \mathrm{~mm}$ and thickness of $0.16-0.19 \mathrm{~mm}$ were analyzed using the same quantifying methods described for the metal coupons. The results were obtained to determine if the methods utilized were reproducible and consistent. Glass coverslips have been used to quantify biofilm formation as it provides a proper surface for confocal microscopy imaging, and it has been shown to be a robust and reproducible method for biofilm evaluation ${ }^{28}$. A typical Z-stacks of CLSM of the biofilm formed on the glass coverslip is shown in Figure 1. 


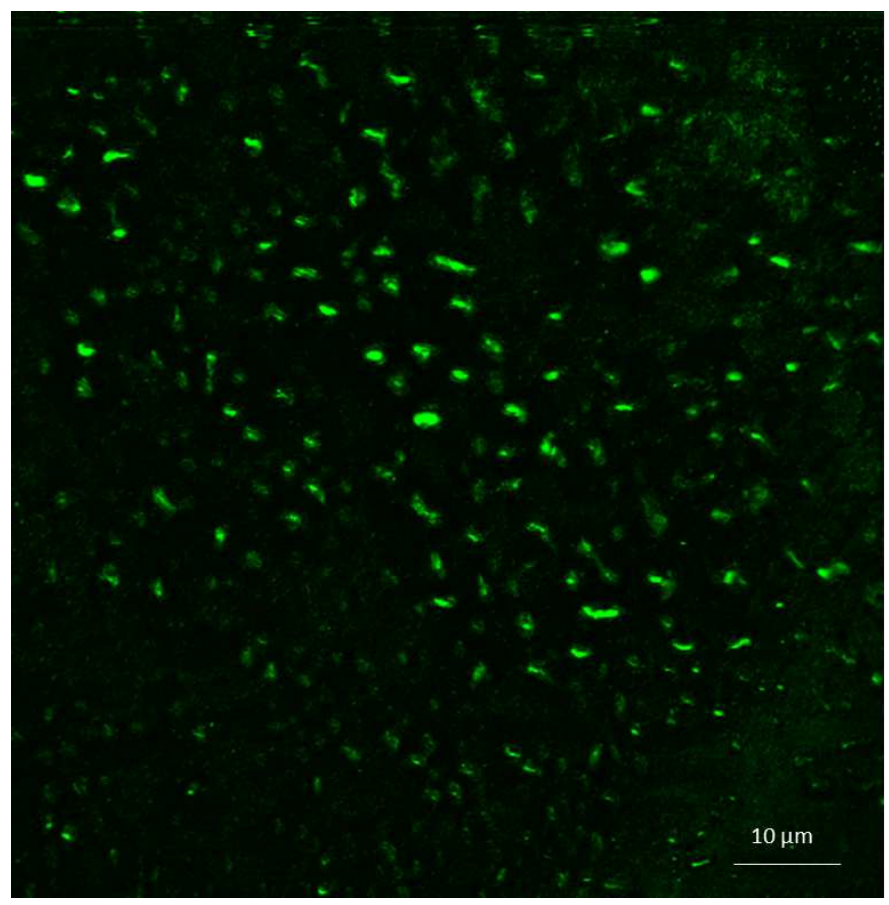

204 Figure 1 A typical Z-stacks of CLSM of the biofilm formed on the glass coverslip. Intensity of green

205 color is proportional to the biofilm presence in the randomly selected location on the glass coverslip

\section{Statistical analysis}

IBM SPSS statistic software (v24) was used for statistical analysis. To analyze the significance of

209 data obtained from individual biofilm quantifying methods, a one-way analysis of variance (ANOVA) test

210 was run with a Tukey post-hoc analysis. The one-way ANOVA test was used to determine whether there

211 were significant differences between the means of data.

\section{RESULTS}

\section{Biofilm quantification results}

The $S$. aureus biofilm formation on individual metal disk surfaces over time was quantified via CV

216 staining, laser confocal microscopy, and viable cell count methods and the results presented as follows. 

was dissolved into ethanol and then the $\mathrm{CV}$ concentration was determined with a microplate reader to 221 estimate the amount of biofilm formed on each surface. The experiment was performed with three 222 replications for each of the Ti and SS coupons. Based on the collected data, no significant difference was 223 observed among the two different coupons. Ti coupons exhibited better biofilm suppression than SS 224 (Figure 2 a).

225

a

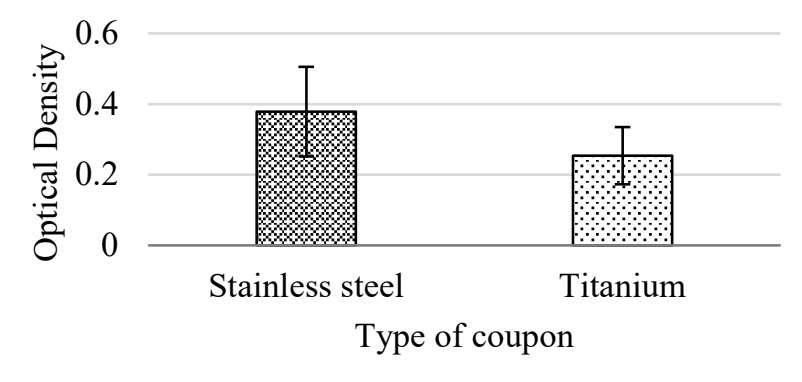

C

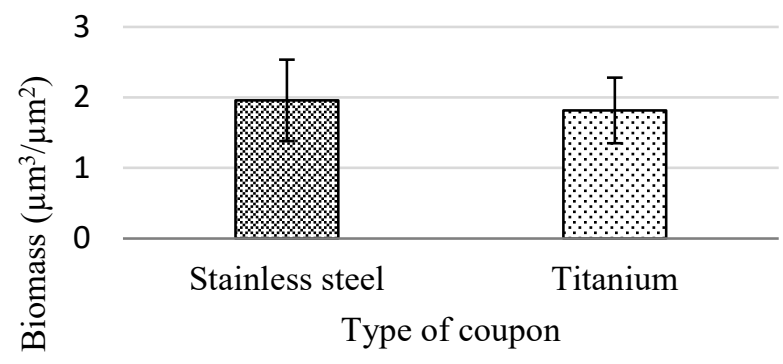

b

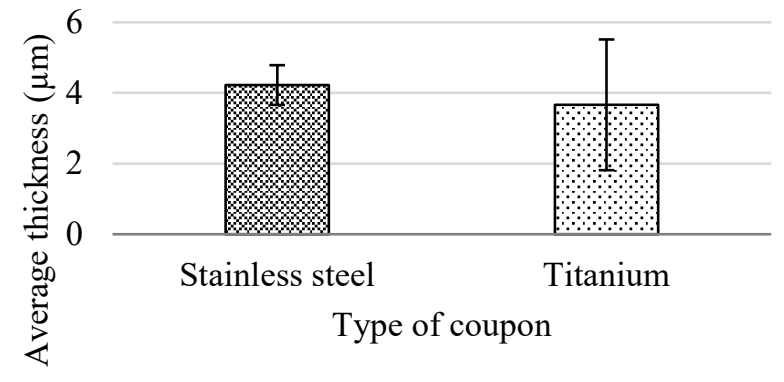

d

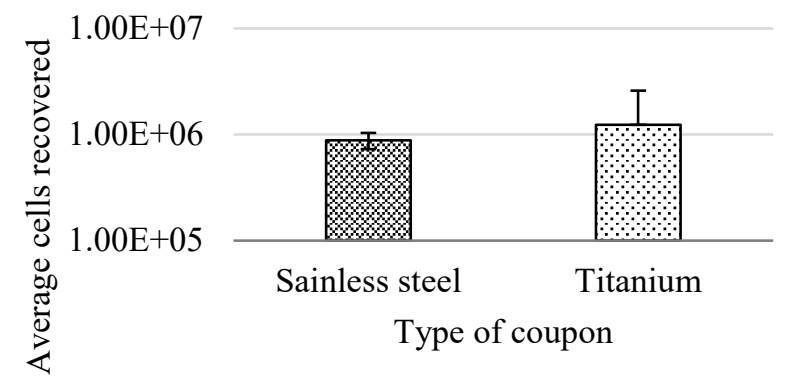

Figure 2 Quantification results of biofilm formed on Ti and SS unmodified coupons a) Crystal violet OD $_{590}$ results of three SS and Ti unmodified coupons. There was no statistical difference between the biofilm growth on the SS and Ti coupons $(\mathrm{n}=3)$; b) Average biofilm thickness of SS and Ti unmodified coupons.

237 There was no statistical difference between the biofilm growth on the SS and Ti coupons (n=3); c) Average biomass on SS and Ti unmodified coupons. There was no statistical difference between the biofilm growth on the SS and Ti coupons ( $\mathrm{n}=3)$; and d) Average cell recovered from SS and Ti unmodified coupons. There was no statistical difference between the biofilm growth on the SS and Ti coupons ( $n=3)$. All statistical analysis was based on t-test two-tailed means comparison analysis 


\section{Analysis of biofilm formation using confocal laser scanning microscopy method}

After bacterial growth on two different surface coupons for $24 \mathrm{hrs}$, the coupons were washed three

244 times with D.I. water to eliminate unattached bacteria from the surfaces. The coupons were then placed into

245 glass bottom chambers upside down while using glass coverslips as spacers to elevate the coupons thus

246 preventing direct contact of biofilms to the bottom of the chamber to avoid changing the biofilm structure.

247 Images were taken using CLSM with a $63 x$ objective and oil immersion. For each coupon, three image 248 stacks were taken in three discrete randomly picked locations. Figure $3(\mathbf{a}, \mathbf{b})$ shows a 3D structure of 249 biofilm on the metal coupon surface, and Figure $3(\mathbf{c}, \mathbf{d})$ shows the wide filed images of biofilm on the 250 metal coupon surface. To analyze the average thickness and biomass of biofilm formed on coupon surfaces, 251 multiple laser confocal image stacks were taken and analyzed using COMSTAT program. From the

252 collected data (Figure $\mathbf{2} \mathbf{b}, \mathbf{c}$ ), Ti and SS material surfaces showed different amounts of biofilm formed on 253 them. However, no significant difference was observed between the material surfaces. 


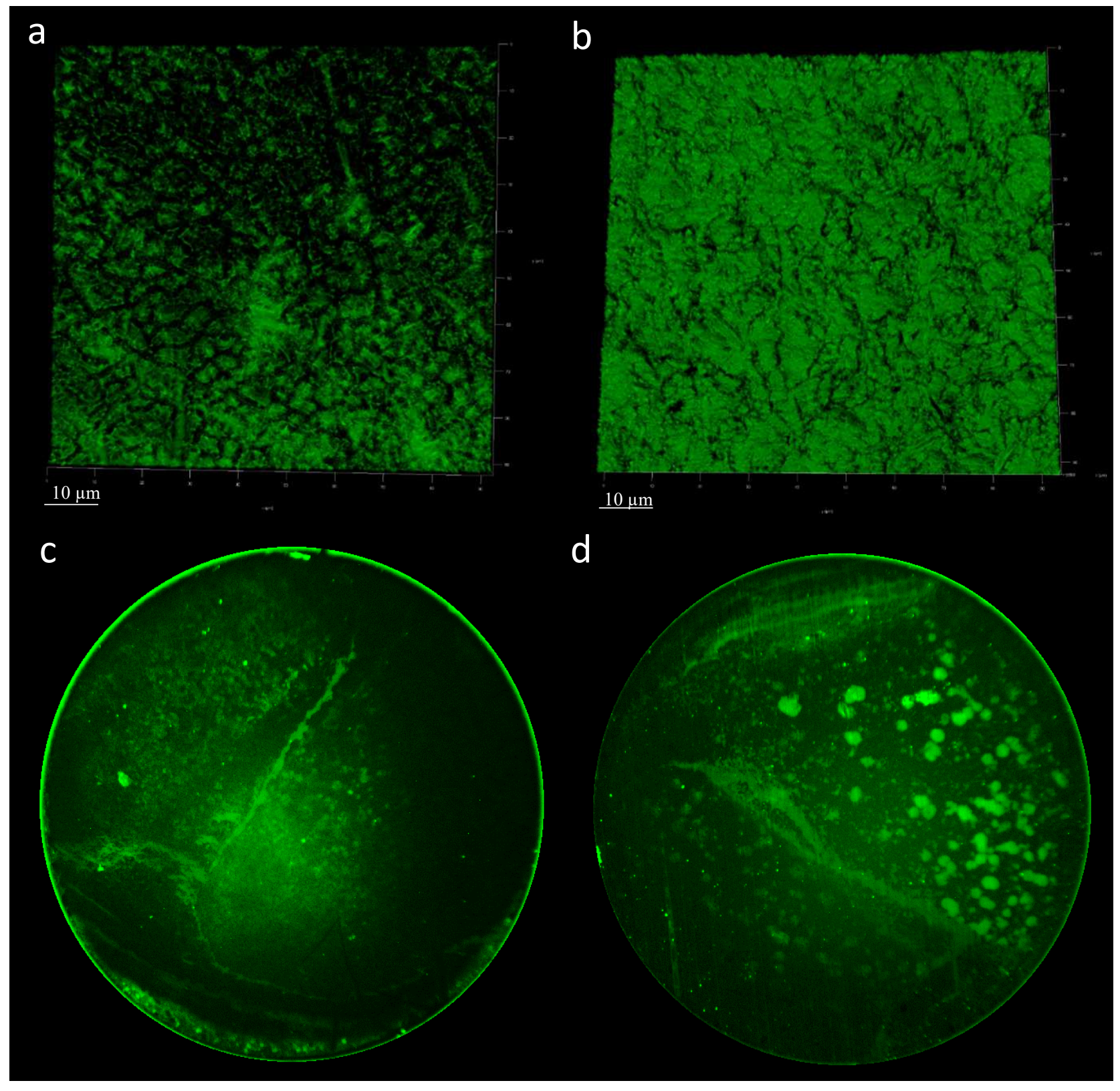

256 Figure 3 3D structures of biofilm on a) SS and b) Ti unmodified coupons. Intensity of green color is

257 proportional to the biofilm present in the randomly selected location of each coupon. Wide-field

258 fluorescence images of biofilm formed on c) SS and d) Ti unmodified coupons diameter equal to $1 \mathrm{~cm}$

259 Analysis of biofilm formation using viable cell count method

260 Evaluation of biofilm on Ti and SS surfaces using viable cell count method indicated that the

261 number of cells recovered from two surface types were not significantly different from each other. Viable 
counts of biofilm recovered from SS and Ti coupons for three complete sets are shown in Figure 2 (d). For both experiments, ANOVA analysis with SPSS software of the collected data did not show significant 264 difference between the SS and Ti coupons $(p=0.05)$.

\section{Comparison of biofilm characterization among methods} different quantifying methods, i.e., CV staining, CLSM, and viable cell count as previously described for the metal coupons. These experiments were conducted to determine whether the used methods are reproducible and consistent in terms of analyzing the amount of biofilm formed on the coupons.

To validate the consistency and reproducibility of the methods, biofilm was grown on the glass coverslips and analyzed using the same three quantification methods used to evaluate biofilm on metal coupons. The results showed that the biofilm formed successfully on the glass coverslips (Figure 4).

275 Quantification of biofilm formed on the glass coverslips demonstrated the consistency and reproducibility 276 of the methods used for enumeration of biofilms. All the data fall within the range of quantified biofilm of 277 the metal coupons. In the CV test, the OD results of three glass coverslips were in the range of $(0.40-0.52)$ 278 with a standard deviation of 0.06 . The viable cell count for total cells recovered from the glass coverslips 279 result fell within (7.59E+05 - 1.21E+06 cells) with a standard deviation of 231902.7. CLSM data showed 280 the biofilm thickness range of $(3.28-4.90 \mu \mathrm{m})$ with a standard deviation of $0.82 \mu \mathrm{m}$. Biomass evaluation 281 of biofilm formed on the glass coverslips were within $\left(1.37-2.23 \mu \mathrm{m}^{3} / \mu \mathrm{m}^{2}\right)$ with a standard deviation of $2820.45 \mu \mathrm{m}^{3} / \mu \mathrm{m}^{2}$. All in all, the obtained data for each different method are close (based on small standard 283 deviation, which indicates that the data points are close to the mean, and $90 \%$ of the obtained data fall 284 within the standard deviation) and this demonstrates the consistency and reproducibility of the methods. 


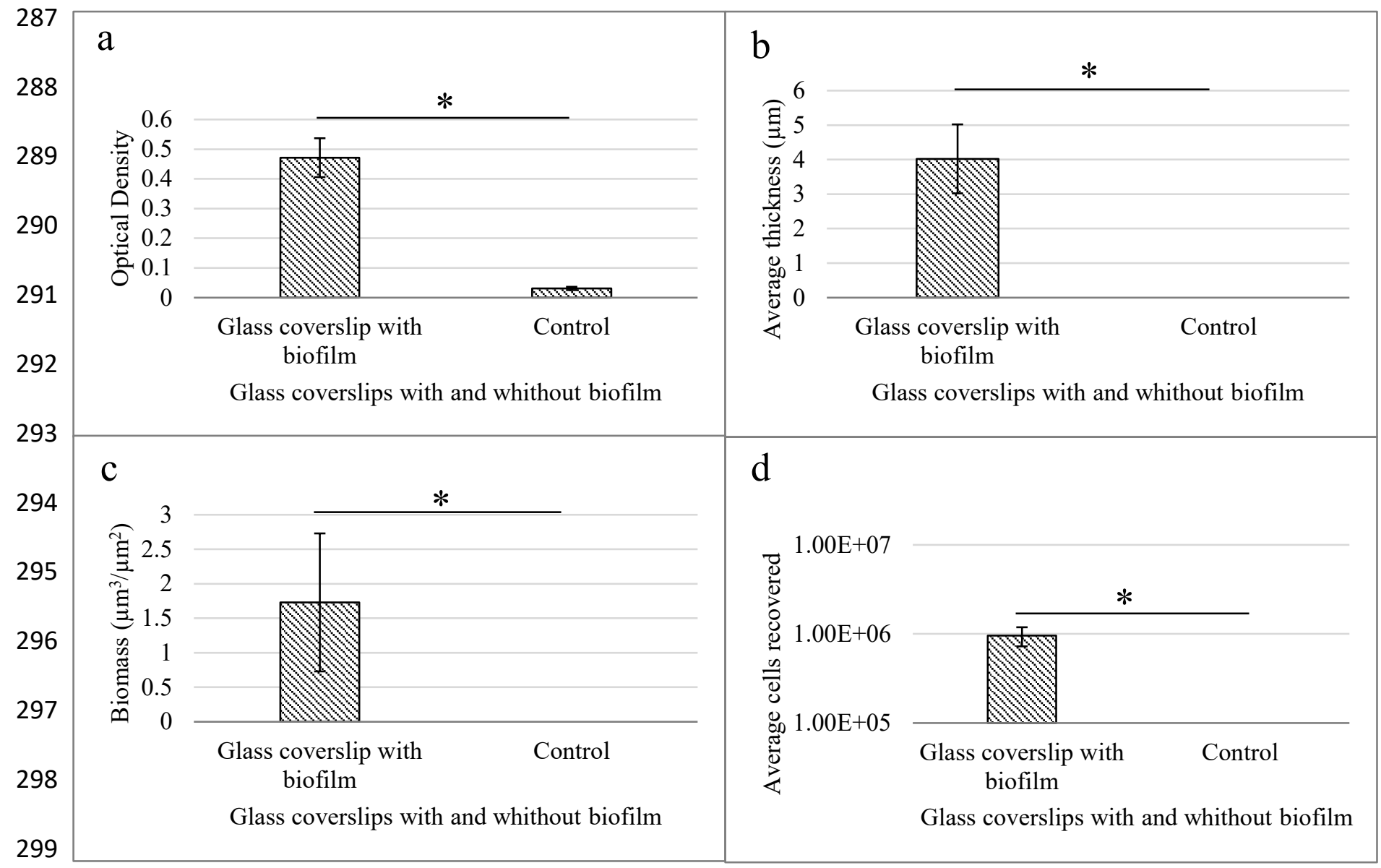

300 Figure 4 Quantification results of biofilm formed on glass coverslip a) Crystal violet $\mathrm{OD}_{590}$ results of three 301 glass coverslips; b) Average biofilm thickness on glass coverslips; c) Average biomass on glass coverslips;

302 and d) Average cells recovered from glass coverslips. * means there is significant difference between two mean groups based on t-test two-tailed means comparison analysis.

\section{DISCUSSION}

Crystal violet is shown to be a suitable staining method for biofilm quantification. Although it can 307 quantify the amount of biofilm on the surface, it stains both live and dead bacterial cells so, cannot 308 determine the functional (live cells) biofilm ${ }^{12,13}$. In comparison with two other biofilm quantifying methods, 309 crystal violet is a quick and simple method best for initial analysis of the modified surfaces. It is sensitive to pipetting and evaporation of the liquids and thus, keeping the experimental conditions consistent for all 
311 samples is challenging and not feasible for a single researcher when evaluation of higher number of samples

312 is needed. For these reasons, using viable cell count method in a larger research study is a more feasible

313 and reliable method for quantification of biofilms. CLSM was the other method used to evaluate biofilm

314 formation. This method enabled us to exam the biofilm structures and biofilm characteristics such as biofilm

315 distribution, thickness and biomass. Although it has been shown that CLSM method provides a detailed

316 evaluation of biofilm, and it can differentiate live and dead cells ${ }^{8}$, this method may not be appropriate for

317 large-scale studies due to the relatively longer time required for each sample analysis and the higher cost

318 associated (both equipment and beam time) when compared to the other methods. Minimum area of at least

$319100,000 \mu \mathrm{m}^{2}$ should also be imaged to have representative data of the biofilm formed on one surface ${ }^{15}$. Each

320 stack area is about $10,000 \mu \mathrm{m}^{2}$ and it takes $30 \mathrm{~min}$ to $1 \mathrm{~h}$ to collect an entire Z-stacks for one area. With at

321 least 10 different Z-stacks of each sample type to be imaged and many different sample types to be

322 evaluated, the required time to complete such task is considerable. In terms of cost comparison, crystal

323 violet and viable cell count method are relatively low-cost methods when compared to CLSM. Viable cell

324 count method can determine the number of live cells in the biofilm and also can be handled better when a

325 large number of samples are being compared at the same time. So, based on the purpose of the work, each

326 of the three different studied methods can be picked to analyze biofilm on orthopedic surfaces. For initial

327 analysis of limited number of samples CV can be used. If time and cost is not a major concern, CLSM is

328 suggested which also can be a proper method for evaluation of the effectiveness of treatments on bacterial

329 viability. When there are a large number of samples to be processed and time and cost are important factors,

330 ultrasound detachment followed by viable cell count is recommended.

\section{Acknowledgments and funding}

This work as financially supported by the Office of the Assistant Secretary of Defense for Health

334 Affairs, through the Peer Reviewed Orthopaedic Research Program under Award No. W81XWH-14-1-

335 0438. Opinions, interpretations, conclusions and recommendations are those of the authors and are not

336 necessarily endorsed by the U.S. Department of Defense. In the conduct of research involving hazardous 

Detrick, MD 21702-5014 is the awarding and administering acquisition office.

\section{Conflict of interest statement}

No conflict of interest declared.

\section{References}

3451 Høiby, N., Bjarnsholt, T., Givskov, M., Molin, S. \& Ciofu, O. Antibiotic resistance of bacterial biofilms. International journal of antimicrobial agents, 322-332 (2010).

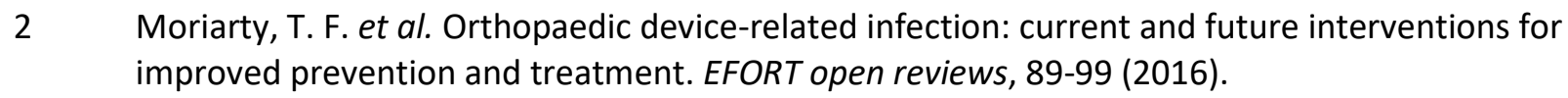

3 Donlan, R. M. Biofilms: microbial life on surfaces. Emerging infectious diseases, 881 (2002).

3504 Nurioglu, A. G., Esteves, A. \& C, C. Non-toxic, non-biocide-release antifouling coatings based on molecular structure design for marine applications. Journal of Materials Chemistry, 6547-6570 (2015).

5 Harris, L. G. \& Richards, R. G. Staphylococci and implant surfaces: a review. Injury 37, 3-14 (2006).

6 Pfaffenroth, C. et al. Self-assembled antimicrobial and biocompatible copolymer films on titanium. Macromolecular bioscience 11, 1515-1525 (2011).

7 Winkel, A. et al. Introducing a semi-coated model to investigate antibacterial effects of biocompatible polymers on titanium surfaces. International journal of molecular sciences, 43274342 (2015).

8 Doll, J., Stumpp, N. S., Winkel, A. \& Stiesch, M. Quantifying implant-associated biofilms: comparison of microscopic, microbiologic and biochemical methods. Journal of microbiological methods, 61-68 (2016).

9 Hayes, J. S. \& Richards, R. G. The use of titanium and stainless steel in fracture fixation. Expert review of medical devices, 843-853 (2010).

10 Christensen, G. D. Adherence of coagulase-negativestaphylococci to plastic tissue culture plates. Clinical microbiology, 996-1006 (1985).

$11 \mathrm{Li}, \mathrm{X}$., Yan, Z. \& Xu, J. Quantitative variation of biofilms among strains in natural populations of Candida albicans. Microbiology, 353-362 (2003).

$12 \mathrm{Xu}, \mathrm{Z}$., Yanrui Liang, Shiqi Lin, Dingqiang Chen, Bing Li, Lin Li, and Yang Deng. Crystal violet and XTT assays on Staphylococcus aureus biofilm quantification. Current microbiolog, 474-482 (2016).

13 Pitts, B., Hamilton, M. A., Zelver, N. \& Stewart, P. S. A microtiter-plate screening method for biofilm disinfection and removal. J. Microbiol Methods, 269-276 (2003).

14 Mueller, L. N., De Brouwer, J. F., Almeida, J. S., Stal, L. J. \& Xavier, J. B. Analysis of a marine phototrophic biofilm by confocal laser scanning microscopy using the new image quantification software. BMC Ecology, 1641-2253 (2006). 
403

404

405

406

407

408

409

410

15 Heydorn, A. et al. Quantification of biofilm structures by the novel computer program COMSTAT. Microbiology, 2395-2407 (2000).

16 Ross, S. S. et al. Quantification of confocal images of biofilms grown on irregular surfaces. Journal of microbiological methods, 111-120 (2014).

17 Heilmann, C., Gerke, C., Perdreau-Remington, F. \& Götz, F. Characterization of Tn917 insertion mutants of Staphylococcus epidermidis affected in biofilm formation. Infection and Immunity 64, 277-282 (1996).

18 Cerca, N., Pier, G. B., Vilanova, M., Oliveira, R. \& Azeredo, J. Influence of batch or fed-batch growth on Staphylococcus epidermidis biofilm formation. Letters in applied microbiology 39, 420-424 (2004).

19 Bjerkan, G., Wits $\varnothing$, E. \& Bergh, K. Sonication is superior to scraping for retrieval of bacteria in biofilm on titanium and steel surfaces in vitro. 80(2), 245-250. Acta orthopaedica, 80, 245-250 (2009).

20 Joyce, A., Al-Hashimi \& Mason, T. J. Assessing the effect of different ultrasonic frequencies on bacterial viability using flow cytometry. journal of applied microbiology, 862-870 (2011).

21 Leid, J. G., E, M., Shirtliff, J., Costerton, W. \& Stoodley, P. Human leukocytes adhere to, penetrate, and respond to Staphylococcus aureus biofilms. Infection and immunity, 6339-3645 (2002).

22 Ham, J.-S. et al. Powerful usage of phylogenetically diverse Staphylococcus aureus control strains for detecting multidrug resistance genes in transcriptomics studies. Molecules and cells, 71-76 (2010).

23 Treangen, T. J. et al. Complete genome sequence of the quality control strain Staphylococcus aureus subsp. aureus ATCC 25923. Genome announcements, 1110-1114 (2014).

24 Bateman, B. T., Donegan, N. P., Jarry, T. M., Palma, M. \& Cheung, A. L. Evaluation of a tetracycline-inducible promoter inStaphylococcus aureus in vitro and in vivo and its application in demonstrating the role of sigB in microcolony formation. Infection and immunity, 7851-7857 (2001).

25 Malone, C. L. et al. Fluorescent reporters for Staphylococcus aureus. Journal of microbiological methods, 251-260 (2009).

26 Jung, I., Powers, T. R. \& Valles, J. M. Evidence for two extremes of ciliary motor response in a single swimming microorganism. Biophysical journal, 106-113 (2014).

27 Russ, J. C. The image processing handbook. (CRC press, 2016).

28 Walker, J. N., and Alexander R. Horswill. A coverslip-based technique for evaluating Staphylococcus aureus biofilm formation on human plasma. Frontiers in cellular and infection microbiology (2012). 


\section{Figures}

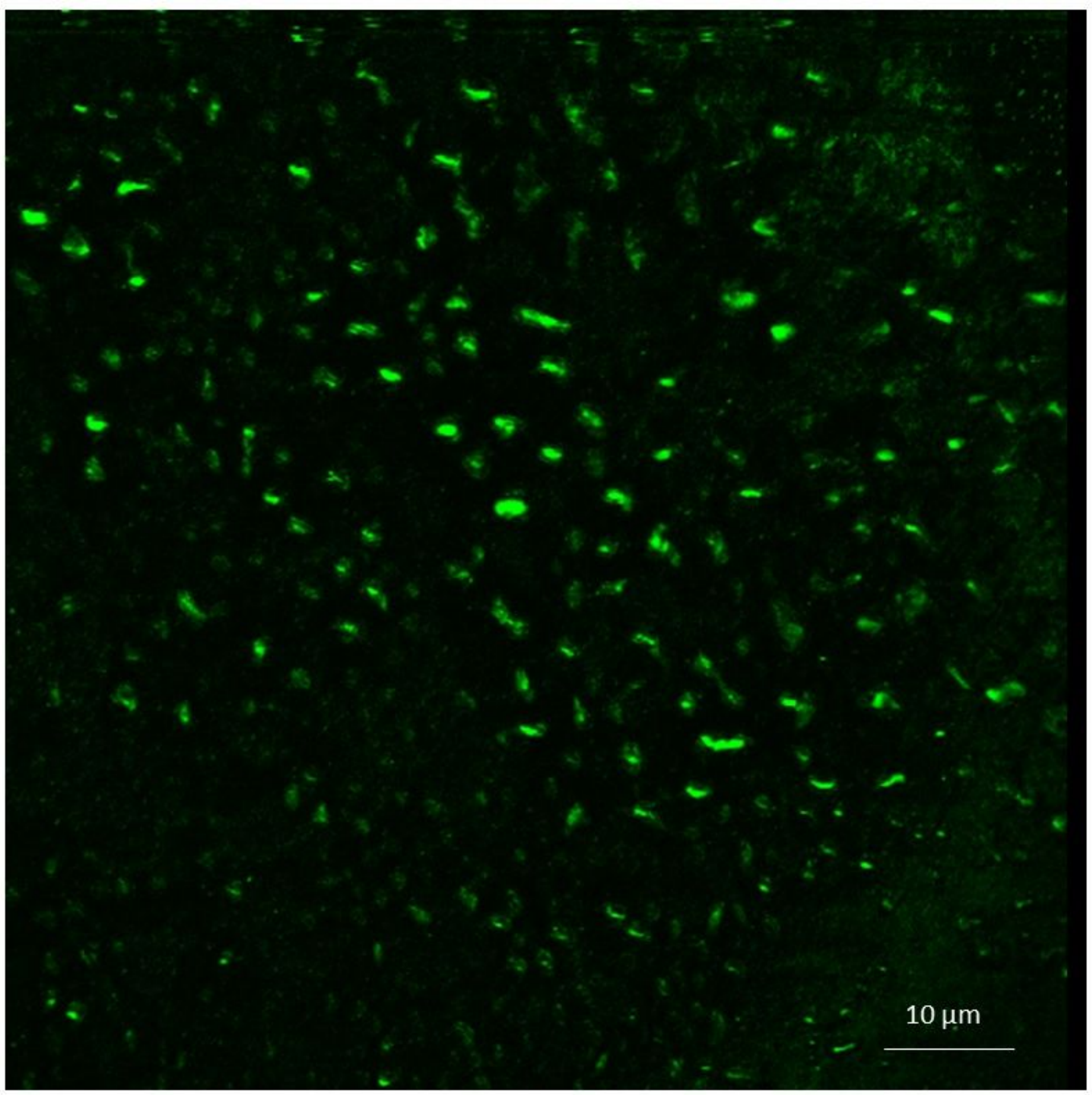

\section{Figure 1}

A typical Z-stacks of CLSM of the biofilm formed on the glass coverslip. Intensity of green color is proportional to the biofilm presence in the randomly selected location on the glass coverslip 


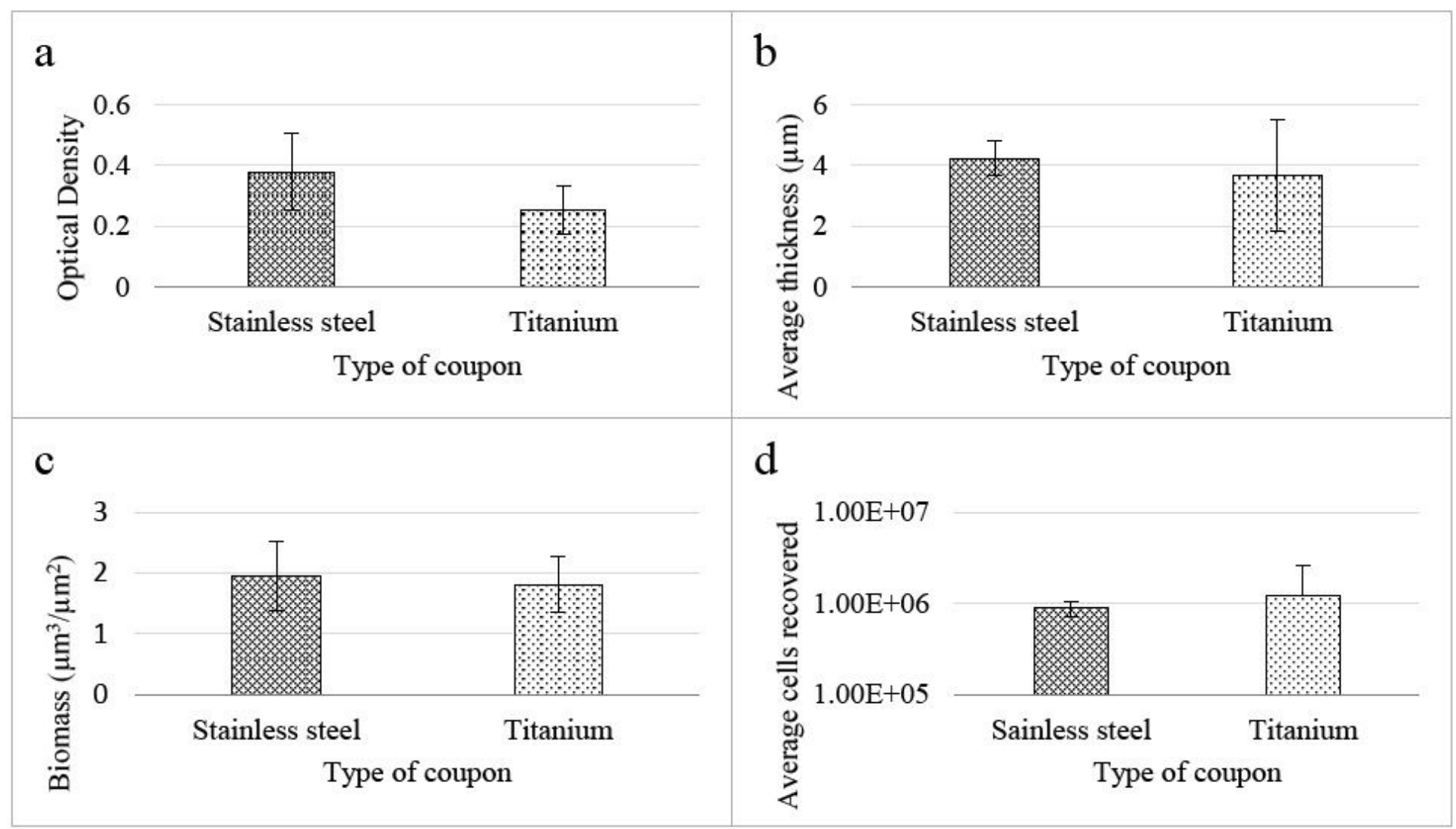

\section{Figure 2}

Quantification results of biofilm formed on Ti and SS unmodified coupons a) Crystal violet OD590 results of three SS and Ti unmodified coupons. There was no statistical difference between the biofilm growth on the SS and Ti coupons ( $n=3) ; b)$ Average biofilm thickness of SS and Ti unmodified coupons. There was no statistical difference between the biofilm growth on the SS and Ti coupons $(n=3) ; c)$ Average biomass on SS and Ti unmodified coupons. There was no statistical difference between the biofilm growth on the SS and Ti coupons ( $n=3)$; and d) Average cell recovered from SS and Ti unmodified coupons. There was no statistical difference between the biofilm growth on the SS and Ti coupons $(n=3)$. All statistical analysis was based on t-test two-tailed means comparison analysis 


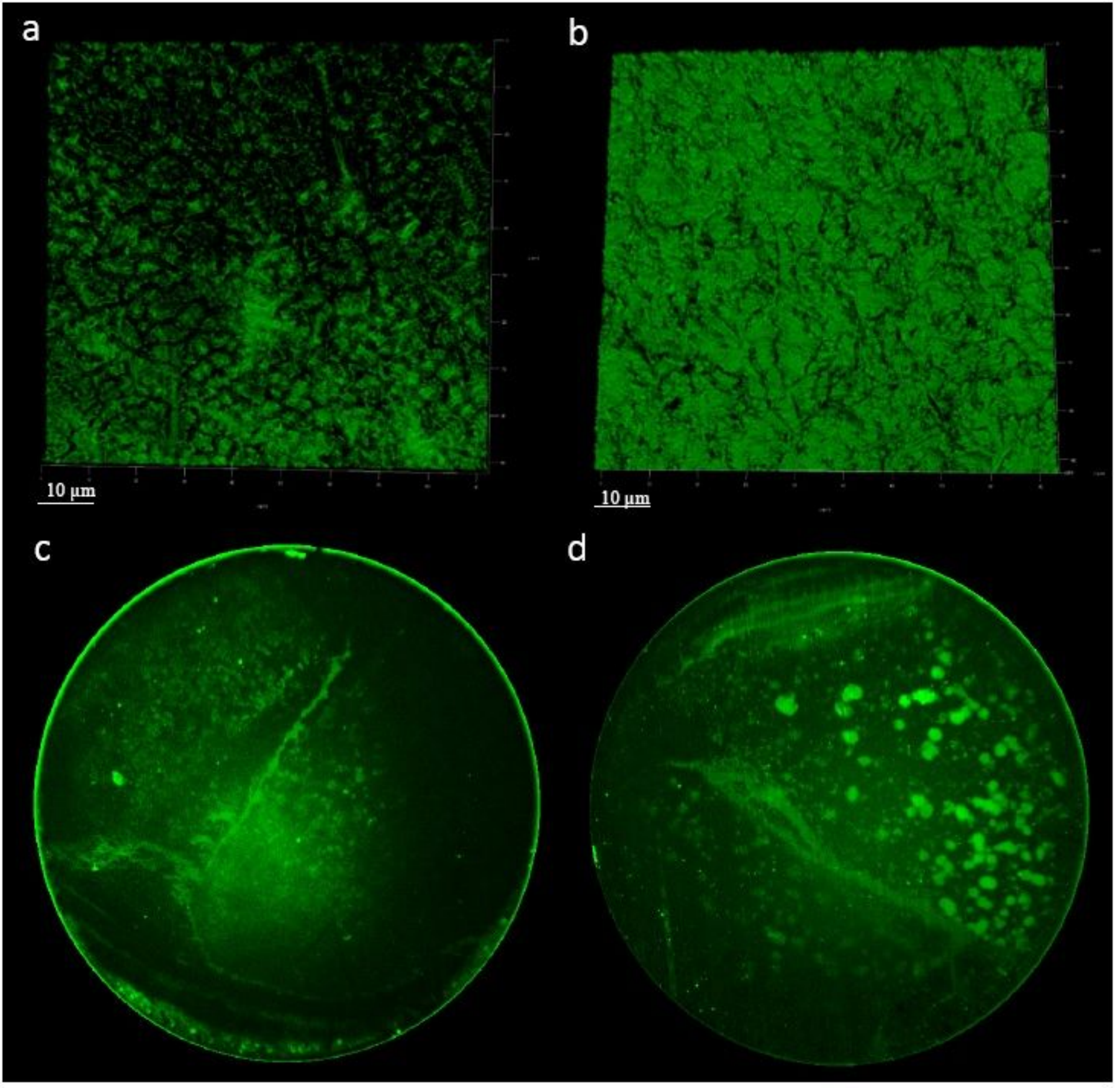

\section{Figure 3}

3D structures of biofilm on a) SS and b) Ti unmodified coupons. Intensity of green color is proportional to the biofilm present in the randomly selected location of each coupon. Wide-field fluorescence images of biofilm formed on c) SS and d) Ti unmodified coupons diameter equal to $1 \mathrm{~cm}$ 


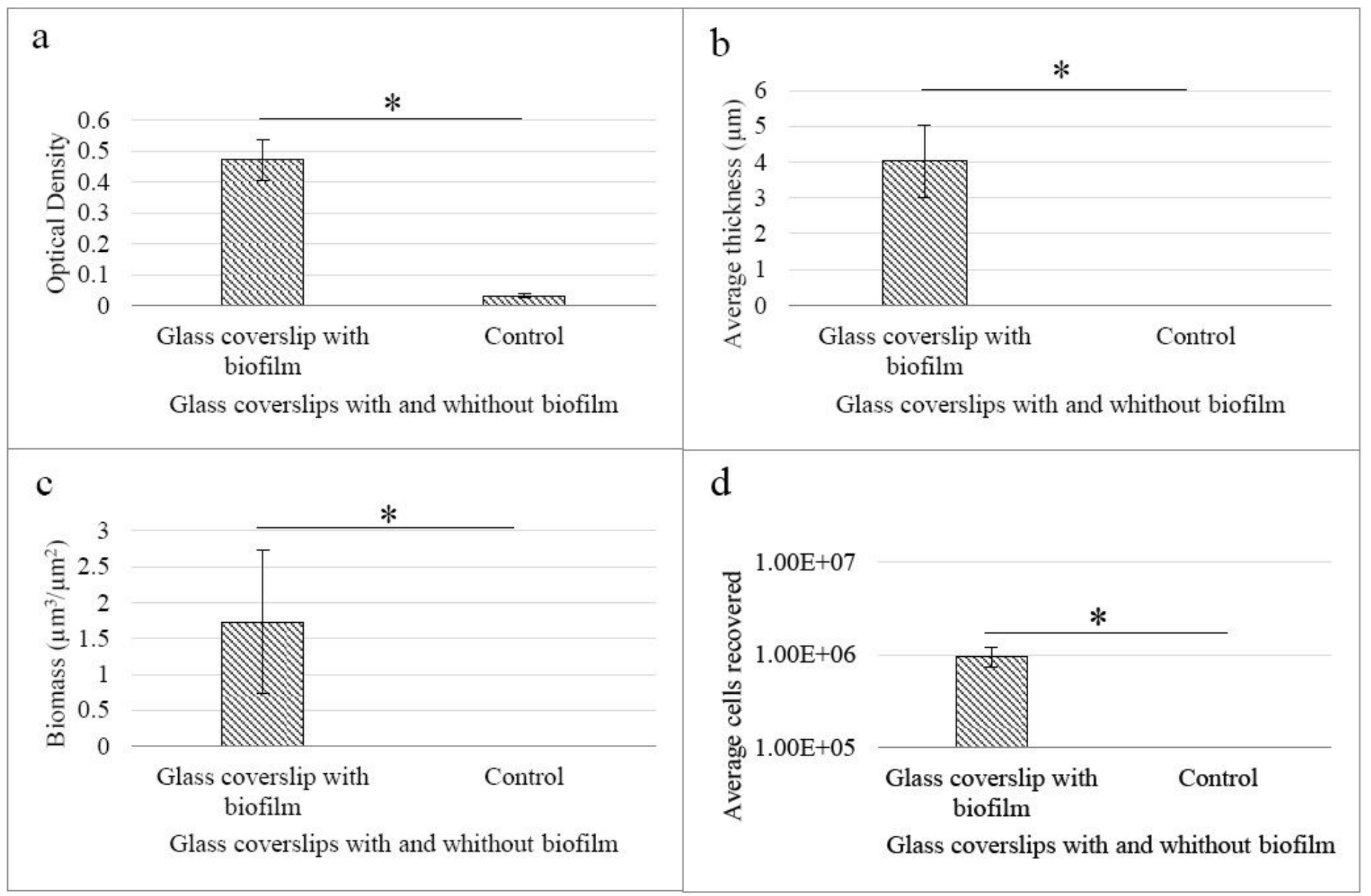

\section{Figure 4}

Quantification results of biofilm formed on glass coverslip a) Crystal violet OD590 results of three glass coverslips; b) Average biofilm thickness on glass coverslips; c) Average biomass on glass coverslips; and d) Average cells recovered from glass coverslips. * means there is significant difference between two mean groups based on t-test two-tailed means comparison analysis. 